\title{
Optimum topology design for the concentrated force diffusion structure of strap-on launch vehicle
}

\author{
Yong $\mathrm{Mei}^{{ }^{*}}$, Guoqiang Liu ${ }^{1}$ and Zebin Shen $^{2}$ \\ ${ }^{1}$ The Third Engineer Scientific Research Institute, Luoyang 471023, China \\ ${ }^{2}$ PLA Foreign Language University, Luoyang 471023, China
}

\begin{abstract}
The thrust from the booster of strap-on launch vehicle is transmitted to the core via the strap-on linkage device, so the reinforced structure to diffusion the concentrated force should be employed in the installation site of this device. To improve the bearing-force characteristics of the concentrated force diffusion structure in strap-on linkage section and realize the lightweight design requirements, topology optimization under multiple load cases is conducted for the concentrated force diffusion structure in this study. The optimal configuration finally obtained can achieve $17.7 \%$ reduction in total weight of the structure. Meanwhile, results of strength analysis under standard load cases show the stress level of this design scheme of the concentrated force diffusion structure meet design requirements and the proposed topology optimization method is suitable for the design of the concentrated force diffusion structure in concept design phase.
\end{abstract}

\section{INTRODUCTION}

Aerospace structures are often assembled from multiple sections. The concentrated force is likely to impact in the installation site of mechanical joints during the external load transferring through the internal structure. The linkage device of the strap-on launch vehicle coupling the core with the booster, and transmits the thrust from the booster to the core. Due to the core and booster employ thin-walled skin structure, which structural forms have small stiffness that is usually necessary to design concentrated force diffusion structure in the installation area of the joints. The force diffusion structure can meet the reliability requirements and transmit the axial or lateral concentrated load from the booster to the core.

The large or heavy launch vehicle with high thrust becoming the focus of research under the development of space technology and demand of the commercial launch, manned lunar landing, deep space exploration [1]. It is reported that a new generation of high-thrust strap-on launch vehicle's booster thrust will reach more than $400 \%$ the maximum thrust of the existing model and the strap-on launch vehicle's core size specification will also be more than twice the existing structure [2]. The traditional strap-on launch vehicle compartment using the skin structure strengthening method which is difficult to bear the large concentration force transferring from the large booster. At the same time that the structure form of solid booster technology may be used in the future changing greatly compared to the existing liquid booster system, so the existing strap-on linkage device structure scheme needs to be redesigned. In addition, the redesigned form should meet the structural stiffness and strength requirements, as far as possible to reduce the mass of concentrated force diffusion structure to realize the lightweight design requirements.

Topology optimization aims to find the best possible layout for a structure within a specified design domain under a set of loads and boundary conditions, which can achieve the optimal performance by changing the topology relation under the premise of satisfying the constraint, and can provide the conceptual design of the structure with the optimal force path in the initial stage of engineering design [3]. Topology optimization has become a very important research in the engineering sector with the tendency of engineering field pursuing lower manufacturing costs, lighter structural weight and better structural performance [4]. It is also concerned with the structural topology with the best performance indexes under the constraints of stress, deformation and other constraints with the deepening of topology optimization study. To this end, carrying out the topology optimization research of concentrated force diffusion surround the typical linkage components bearing-force characteristics in the aerospace structure, especially in the launch vehicle structure is very urgent.

This study carries out the conceptual design for the concentrated force diffusion structure based on the load index and structure specification of a certain type highthrust strap-on launch vehicle. Firstly, the finite element model of the initial structure design is built by using MSC.PATRAN/ NASTRAN, and the simulation analysis is carried out under the standard load case to

\footnotetext{
Corresponding author: meiyong1990@qq.com
} 
determine the topology optimization area. Then, based on the FE-DESIGN company's TOSCA software platform, the topology optimization for the concentrated force diffusion structure is carried out by using the minimizing weighted compliance of the multiple load cases as the objective function, the manufacturing constraints and the volume ratio as the constraint conditions. Finally, a new type of concentrated force diffusion structure is designed and the intensity checking is carried out according to the topology optimization results.

\section{MATHEMATICAL MODEL OF TOPOLOGY OPTIMIZATION PROBLEM UNDER MULTIPLE LOAD CASES}

Solid isotropic material with penalization (referred to as SIMP) is the most commonly used continuum structure topology optimization method. The relative density of the element is usually designated as the design variable and generally $(0,1)$ distributed in the initial design area after topology optimization [5]. The interpolation model of SIMP algorithm mostly uses density-stiffness exponential model which assumes that the elastic modulus of the material and its density have the following relation:

$$
\left\{\begin{array}{l}
\rho(x)=x_{e} \rho_{0} \\
E(x)=x_{e}^{p} E_{0}
\end{array}\right.
$$

Where $x_{e}$ is the relative density of the element, $\rho(x)$ is the design variable of topology optimization, $\rho_{0}$ is the initial density of the element in the design region, $\mathrm{E}(x)$ is the elastic modulus after optimization, $E_{0}$ is the initial elastic modulus, $p$ is the penalty factor.

Before the separation of the strap-on launch vehicle booster and the core, it needs to focus on the load case in several typical states of the concentrated force diffusion structure. The minimum compliance of the structure under different load cases is different for the topology optimization problem with the objective of minimizing the compliance. In order to ensure that the compliance of the structure is as small as possible under multiple load cases, Gong [6] uses the weighted average of the structural compliance under each load case as the optimal compliance of the structure, and the original optimization problem is transformed to multi-objective optimization problem. Thus, the topological optimization model of multiple load cases can be established based on the SIMP algorithm with the objective of minimizing the structural weighted average of mean compliance $C$ and the volume ratio $\alpha$ as the constraint:

$$
\begin{aligned}
& \underset{\rho_{1}, \cdots, \rho_{2}}{\min } \boldsymbol{C}= \sum_{i=1}^{l} \eta_{i} \boldsymbol{C}^{i}=\sum_{i=1}^{l} \eta_{i} \boldsymbol{U}_{i}^{T} \boldsymbol{K} \boldsymbol{U}_{i} \\
& \text { subject to }\left\{\begin{array}{l}
\boldsymbol{F}=\boldsymbol{K} \boldsymbol{U} \\
\sum_{j=1}^{n} V_{j} \rho_{j}-V^{*} \leq 0 \quad V^{*}=\alpha V_{0} \\
0<\rho_{\min } \leq \rho_{j} \leq 1 \quad j=1,2, \cdots, n
\end{array}\right.
\end{aligned}
$$

Where $l$ is the total number of load cases, $\eta_{i}$ is the weight factor of the ith condition, $\boldsymbol{C}^{i}$ is the compliance of the $i$ th condition, $\boldsymbol{C}$ is the weighted compliance of the multiple load cases, $\boldsymbol{K}$ is the total stiffness matrix , $\boldsymbol{F}$ is the load vector, $\boldsymbol{U}$ is the displacement vector, $\alpha$ is the volume ratio, $V_{0}$ is the initial volume of the design domain material, $V^{*}$ is the volume of the material allowed, $V_{j}$ is the volume of the element, $\rho$ is the density of the element, $\rho_{\min }$ is the lower limit value of the design variable and generally in order to avoid the total stiffness matrix singular, take $\rho_{\min }=0.001, n$ is the number of elements.

\section{FINITE ELEMENT MODELING AND ANALYSIS OF CONCENTRATED FORCE DIFFUSION STRUCTURE}

\subsection{Introduction of concentrated force diffusion structure}

For the strap-on launch vehicles, the booster's gravity and the engine thrust will be transmitted to the core through the strap-on linkage device and the installation area of the linkage device is the part of concentrated force applied. The concentrated force diffusion structure of the shell is usually designed as the main structure for the bearing capacity, which using reinforced bearing and joint structure to diffusion concentration corresponding to the concentrated force of the part.

Concentrated force diffusion structure consists of the front and rear frame components, three intermediate frame, skin, composite beam, vice beam, reinforced bearing, joint and stringer (the design of concentrated force diffusion structure in this study does not consider the import and export hole of cable). The whole structure is symmetrical by the quadrant. Where reinforced bearing and joint structure using 7A09 aluminum alloy, the rest of the structure using 2A14 aluminum alloy material, the relevant material parameters shown in Table 1.

Table 1. Material property parameter

\begin{tabular}{|c|c|c|c|c|}
\hline Material & $\begin{array}{c}\text { Elastic } \\
\text { modulus }\end{array}$ & $\begin{array}{c}\text { Poisson's } \\
\text { ratio }\end{array}$ & $\begin{array}{c}\text { Yield } \\
\text { limit }\end{array}$ & $\begin{array}{c}\text { Strength } \\
\text { limit }\end{array}$ \\
\hline $2 \mathrm{~A} 14$ & $70560 \mathrm{MPa}$ & 0.3 & - & $355 \mathrm{MPa}$ \\
\hline $7 \mathrm{~A} 09$ & $70560 \mathrm{MPa}$ & 0.3 & $315 \mathrm{MPa}$ & $500 \mathrm{MPa}$ \\
\hline
\end{tabular}

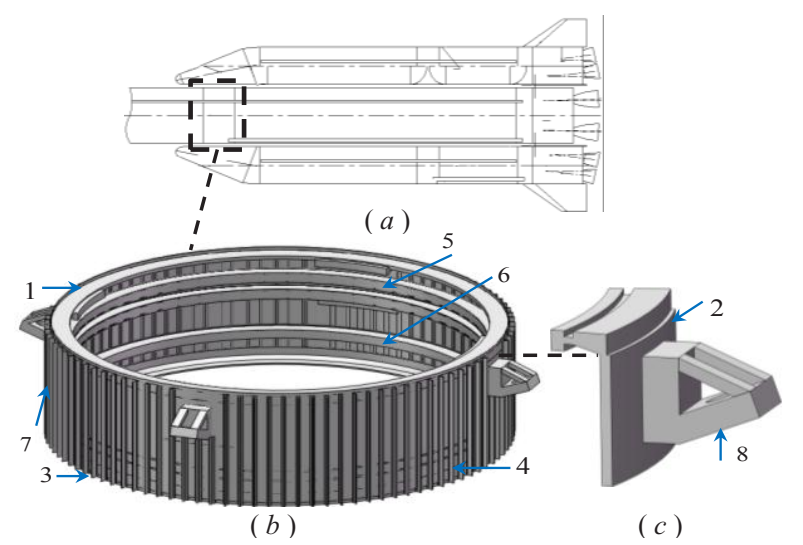


1. front frame, 2. reinforced bearing, 3. rear frame, 4. stringer,

5. front intermediate frame, 6 . rear intermediate frame, 7. skin, 8. joint

Fig. 1. The concentrated force diffusion structure

\subsection{Finite element model of concentrated force diffusion structure}

The load cases and the corresponding load value of the concentrated force diffusion structure in mechanics analysis are determined according to the analysis results of the early strap-on launch vehicle transmission path. Since the boosters are generally symmetrically mounted around the core, $1 / 4$ finite element model of concentrated force diffusion structure built based on MSC.PATRAN can be used for the analysis. In this study, the front and rear ends of concentrated force diffusion structure connects with an extended section, which is set to be elastic supporting region as non-design domain considering the connection stiffness of the structure in engineering practice. The boundary conditions on the top of front extension and bottom of rear extension are set to be fixed, and the extension section is connected to the main structure by means of the nodes equivalent. In addition, the symmetry boundary conditions in the cylindrical coordinate system are applied to both left and right sides of the concentrated force diffusion structure. The finite element model shown in Figure.2 (a) is modeled by Hex 8 entity element with a total of 260504 elements, 345223 nodes, and the influence of the skin is not considered during the analysis.

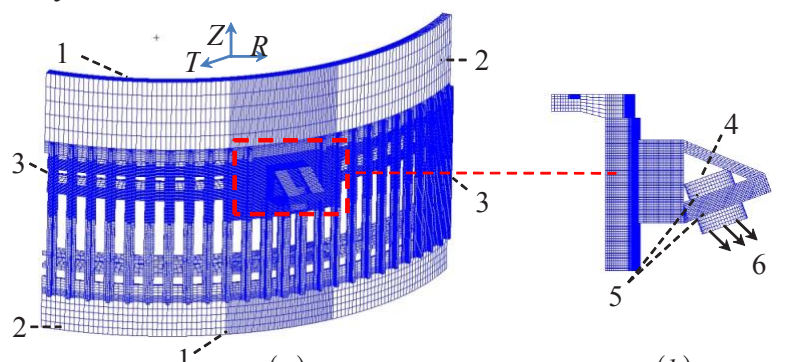

(a)

(b)

1. fixed support, 2. extended section, 3 . symmetric boundary condition, 4. strap-on linkage device, 5. contact interface, 6. load

Fig. 2. The FEA model of the concentrated force diffusion structure

In this study, the actual assembly relation between the strap-on linkage device and the concentrated force diffusion structure is simplified, which is equivalent to contact and the contact type between the device and the joint defined as flexible contact, the load transmitted by the booster is loaded on the end of joint on the strap-on linkage device, other boundary conditions processing methods are shown in Figure 2 (b). The strength analysis of the concentrating force diffusion structure on strap-on launch vehicle under multiple load cases is carried out using the linear contact solver 101 of NASTRAN. The strength analysis in the conceptual design phase requires consideration of the 4 standard conditions shown in Table 2, requiring that the maximum Von Mises stress of the structure does not exceed the strength limit of the associated material and the maximum deformation is less than $15 \mathrm{~mm}$.

Table 2. Analysis load cases

\begin{tabular}{|c|c|c|c|c|l|}
\hline Load & time/ & \multicolumn{3}{|c|}{ load $/ \mathrm{kN}$} & \multicolumn{1}{|c|}{ remarks } \\
\cline { 3 - 4 } case & $\mathrm{s}$ & $Z$ & $R$ & $T$ & \\
\hline 1 & -5.0 & 235 & -296 & 0 & Loaded with fuel \\
\hline 2 & $0+$ & 688 & -89 & 0 & Take off \\
\hline 3 & 149 & 1860 & -94 & 0 & $\begin{array}{l}\text { Reach the maximum } \\
\text { acceleration }\end{array}$ \\
\hline 4 & 150 & -214 & 27 & 0 & $\begin{array}{l}\text { Turn off the booster } \\
\text { engine }\end{array}$ \\
\hline
\end{tabular}

\subsection{Finite element analysis results of concentrated force diffusion structure}

The strength analysis results of concentration force diffusion structure under multiple load cases are shown in Table 3. The results show that the initial design configuration satisfies the structural stiffness and strength design requirements, and the maximum Von Mises stress is much smaller than the material strength limit, so in this study will carry out topology optimization based on this design configuration.

Table 3. The structural static response under the typical load cases

\begin{tabular}{|c|c|c|}
\hline $\begin{array}{c}\text { Load } \\
\text { case }\end{array}$ & $\begin{array}{c}\text { Maximum Von Mises } \\
\text { Stress/ MPa }\end{array}$ & $\begin{array}{c}\text { Maximum } \\
\text { deformation/ mm }\end{array}$ \\
\hline 1 & 55 & 0.5 \\
\hline 2 & 131 & 3.0 \\
\hline 3 & 375 & 8.6 \\
\hline 4 & 41 & 0.9 \\
\hline
\end{tabular}
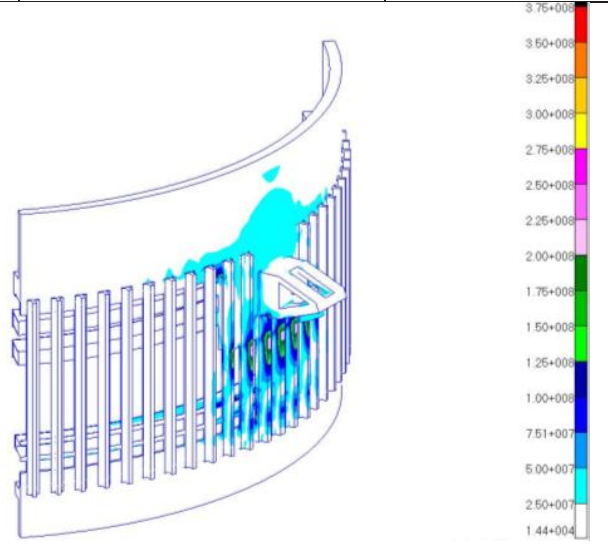

Fig. 3. Stress nephogram of the concentrated force diffusion structure

It can control the optimization and non-optimization parts of the structure by specifying the design and nondesign domain in the topology optimization. Figure.3 shows the Von Mises stress nephogram of the concentrated force diffusion structural of strength analysis under load case 3 . The results of the stress distribution show that the maximum stress concentrated in the reinforced bearing and joint (the conclusion of 1, 2, 4 load cases is the same as that described in case 3 ). In addition, in order to strengthen the reinforced bearing and joint using a large number of materials in the initial design which quality accounts for $33 \%$ of the total 
weight. There is an urgent need for weight reduces in the realization of lightweight design while changing the original concentration form of force diffusion. Therefore, the front frame, intermediate frame, rear frame, etc. are designated as non-design domain and reinforced bearing, joint are set to be optimize design domain. At the same time that the manufacturing constraints of the symmetrical distribution are defined based on the centerline of the structure.

\section{OPTIMUM DESIGN AND RESULT CHECK OF CONCENTRATED FORCE DIFFUSION STRUCTURE}

\subsection{Optimum design of concentrated force diffusion structure}

Based on Tosca software platform and integrate Nastran solver for optimum topology design of the concentrated force diffusion structure, and the SIMP algorithm is carried out by using the method of moving asymptotes algorithm (referred to as MMA) to iterative solution of the optimization model. In the conceptual design stage set 4 conditions are equally important and the weight factor $\eta_{i}$ in the formula (2) is processed according to the linear weighted method. In TOSCA system set the compliance weight factor of each condition is $25 \%$ to the total compliance, so as to obtain the weighted average structure compliance under multiple load cases.

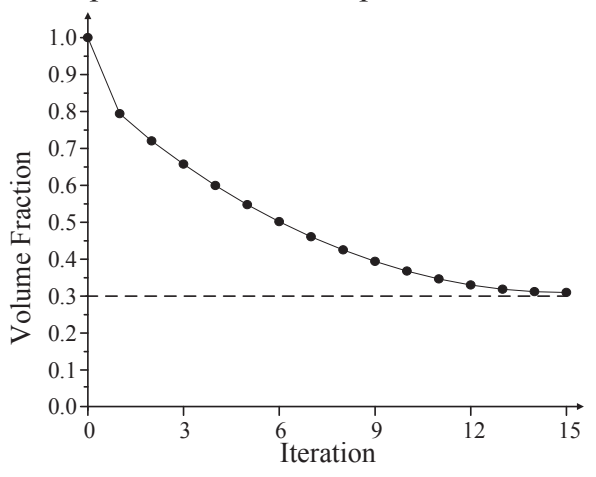

Fig. 4. Evolution history of volume fraction

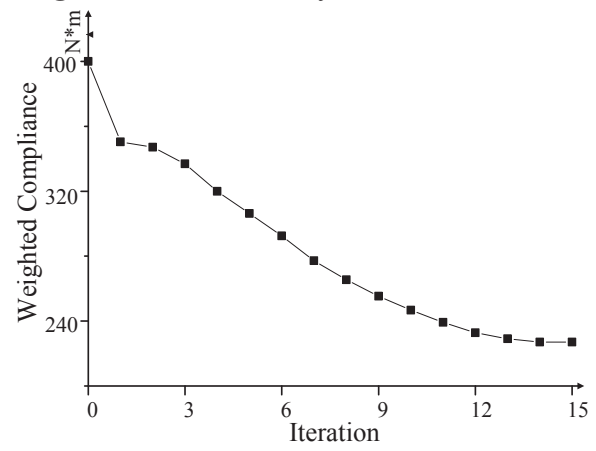

Fig. 5. Variable trend of the weighted compliance

The optimum topology design of the structure is carried out by taking the minimum weighted compliance for the target volume of $30 \%$ of the initial design domain as the objective, considering the performance of concentrated force diffusion and the stiffest design as the constraints based on the SIMP algorithm. Figure 4 shows the evolution history of volume fraction for the reinforced bearing and joint structure under 4 load cases. It can be seen that a stable material removal was carried out through the SIMP optimization process after 15 iterations and the volume ratio is approximately $30 \%$ of the initial design domain. The iteration history of the weighted compliance is shown in Figure 5.

The optimal material distribution results of the reinforced bearing and joint structure after topology optimization and boundary smoothing within the design domain are shown in Figure. 6. The density of the whole topology optimization result is basically symmetrical distribution and achieving a smooth solution.

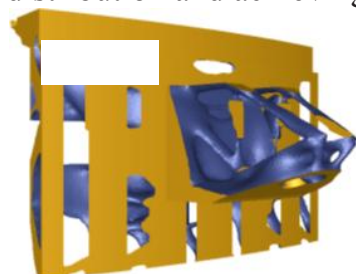

(a)
( $b$ )

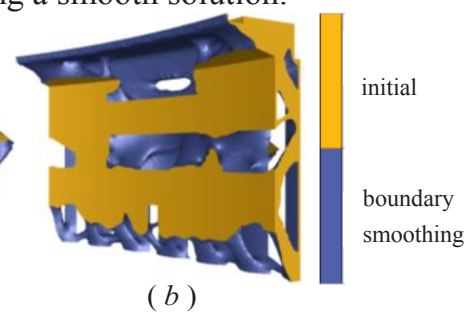

Fig. 6. Optimal topological structure of support and joint

4.2 Re-modeling and result check of the new concentrated force diffusion structure

It is necessary to redesign the reinforced bearing and joint structure on the basis of the engineering practice according to the optimum topology configuration. The main considerations are as follows: (1) If a density based method, like SIMP, is employed for topology optimization, there is a need to threshold at an arbitrary density to achieve a discrete solution which contains some parts of intermediate relative density material that is not present in the actual structure and should be modified to improve the optimality of the solution; (2) the shape of the material-filled region boundary is more complex or even discontinuous in the material distribution of the optimum topology design, which is inconsistent with the actual structure design rules at the same time increasing the processing difficulty. So it is necessary to simplify the optimal topological configuration and require additional post-processing, such as smoothing and shape optimization, to generate a manufaturable design with smooth and clearly defined boundaries. The redesigned reinforced bearing and joint structure are shown in Figure 7 out of consideration of these circumstances, the results showed a significant improvement in boundary representation and structural performance of the solutions. Compared with the initial configuration shown in Figure.1 (c), the material at the top of the reinforced bearing and joint is partially cut. The whole structure of new reinforced bearing and joint is integrally trapezoidal. The new structure will result in a $17.7 \%$ reduction in the overall concentrated force diffusion structure and achieve the goal of lightweight design. 


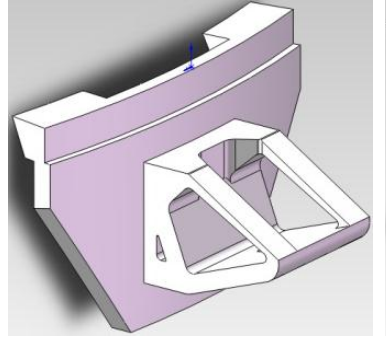

(a)

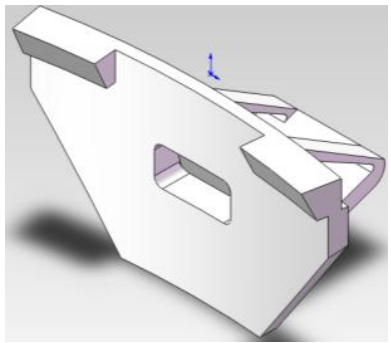

(b)
Fig. 7. The new support and joint according to the optimum topology design

In order to verify whether the designed newly concentrated force diffusion structure can meet the requirements of structural strength and stiffness, the newly designed structures are discretized and meshed by finite element replacing the original structures which have defined material parameters and element properties. Define the contact boundary conditions and deformation constraints between the various components, and perform the strength analysis under the standard load cases as with the previous analysis.

The calculation results of the maximum Von Mises stress and deformation under 4 typical load cases are shown in Table 4. The maximum stress and deformation appear in the case 3, the corresponding maximum stress is $466 \mathrm{MPa}$ which is less than the material strength limit, and the maximum deformation is $11 \mathrm{~mm}$ which is still less than the stiffness requirement in the concept design. Therefore, the maximum stress and the deformation value of the redesigned concentrated force diffusion structure according to the topology optimization results conform to the design index of the stiffness and strength in the conceptual design stage. So the structure design scheme is reasonable.

Table 4. Optimized design results

\begin{tabular}{|c|c|c|}
\hline $\begin{array}{c}\text { Load } \\
\text { case }\end{array}$ & $\begin{array}{c}\text { Maximum Von Mises } \\
\text { Stress } / \mathrm{MPa}\end{array}$ & $\begin{array}{c}\text { Maximum } \\
\text { deformation } / \mathrm{mm}\end{array}$ \\
\hline 1 & 84 & 0.9 \\
\hline 2 & 158 & 3.9 \\
\hline 3 & 466 & 11.0 \\
\hline 4 & 55 & 1.2 \\
\hline
\end{tabular}

\section{CONCLUSIONS}

In this study, take the minimum weighted compliance of the structure as the objective function, considering the performance of concentrated force diffusion and the stiffest design for the target volume of $30 \%$ of the initial design domain as constraint, the optimum topology design of the concentrated force diffusion structure is carried out based on the SIMP algorithm in the optimization software TOSCA integrating finite element analysis software MSC.PATRAN/ NASTRAN.

The additive manufacturing design of the structure is carried out according to the topology optimization results. The weight of new concentrated force diffusion structure configuration is $17.7 \%$ less than the original ones, and the maximum stress and deformation of the structure under specific multiple load cases are lower than those in the conceptual design stage. Finally, the goal of lightweight design is realized.

Consider the fact that this study only considers the structural optimization under static load cases. The results can be more in line with the engineering requirements if the topology optimization taking into consideration under dynamic response of the flight process.

\section{References}

1. L.H. Long, X.J. Wang, Y. Rong, The development prospect of expendable launch vehicle in China. Chinese science E: technical science, 39, (2009), 460-463.

2. W. He, W. Liu, G. Li, Heavy launch vehicle and its application. Missile and space vehicle, 1, (2011), 15.

3. X.H. Wang, H.F. Zheng, H.P. Liu, et al, Structural optimization of folding wing based on HyperWorks. Journal of solid rocket technology, 6, (2012), 795798.

4. A.R. Yildiz, Comparison of evolutionary- based optimization algorithms for structural design optimization Engineering applications of artificial intelligence, 1, (2013), 327-333.

5. M.P. Bendsoe, O. Igmund, Material interpolation schemes in topology optimization, Archive of Applied Mechanics, 69, (1999), 635-654.

6. S.G. Gong, X. Liu, G.L. Xie, et al, Topology optimization under multi-load cases based on element-free Galerkin method, Journal of mechanical engineering, 12, (2009), 137-14 